\title{
ANGULAR DISTRIBUTIONS OF SECONDARY ELECTRONS EMITTED FROM THE POLYCRYSTALLINE SILVER
}

\author{
W. Dolińsiki, J. Palczyńsiri, P. Mazur and L. Gieniec \\ Institute of Experimental Plyysics, University of Wroclaw \\ Cybulskiego 36, 50-205 Wrocław, Poland \\ (Received June 14, 1991; in revised form July 22, 1991)
}

The angular distributions of secondary electrons (including Auger electrons) of different energies emitted from polycrystalline silver bombarded with primary electrons $\left(E_{\mathrm{p}}=1000\right.$ and $\left.2500 \mathrm{eV}\right)$ were measured with a RFA analyzer equipped with an additional collector (acceptance angle $\approx 4^{\circ}$ ). Additionally, the angular distributions of primary electrons of different energies elastically backscattered from the same sample were also investigated by means of a specially constructed rotable RFA analyzer equipped with a channeltron. The results obtained show the cosine distribution of secondary and Auger electrons emitted as shown previously in the literature. The angular distributions of electrons backscattered elastically depend on electron energy and are in good agreement with theoretical calculations (Monte Carlo simulation of primary electrons trajectories in solids) proposed by Jabloński.

PACS numbers: 79.20. $\mathrm{Hx}, 79.20 . \mathrm{Fv}, 34.80 . \mathrm{Bm}$

\section{Introduction}

The information about the shape of the angular distribution of secondary electrons emitted from a given sample bombarded with primary electrons can be important for the calculations of the total current of electrons emitted. Such problem appeared among others in our previous paper [1] where the so-called backscattering factor (an important factor for quantitative Auger and X-ray microanalysis) was determined for noble metals and their alloys. The calculations of total electron current (emitted in the range of angles $0^{\circ}-90^{\circ}$ in respect to surface normal) were done in Ref. [1] based on the measurements of secondary electron energy distribution $N(E)=f(E)$ measured with an RFA analyzer and the assumption of a cosine distribution of secondary electrons independent on the electron energy as proposed by Gerlach and Ducharme [2]. However, other authors have reported different results concerning the angular distributions of secondary electrons. For example in the papers of Jonker [3], Bruining [4] and Kanter [5] the same cosine character of 
secondary electron angular distribution was reported and additionally the inverse proportionality of secondary electron yield to the incidence angle of the primaries was found. However, in the papers of Pendry [6] and Aberdam et al. [7] the cosine square distribution was predicted theoretically for Auger electrons. Shimizu and Ichimura [8] have found once more the cosine distribution of secondary electrons based on Monte Carlo simulation of electron trajectories in the solids, but the experimental results obtained by Oguri et al. [9] for Auger electrons ( $\mathrm{Cu}-\mathrm{M}_{2,3} V V$ Auger line) and secondary electrons $\left(E \approx 10 \mathrm{eV}, E_{\mathrm{p}}=300 \mathrm{eV}\right)$ show neither cosine nor cosine square distribution. It seems that the problem needs some more investigations, and this was the reason for us to measure the angular distributions of secondary electrons $(E=50,100$ and $400 \mathrm{eV})$ and also Auger electrons (Ag-MNN Auger line, $E_{\mathrm{p}}=2500 \mathrm{eV}$ ) emitted from polycrystalline silver. Another problem is connected with angular distributions of electrons backscattered elastically. In 1985 Jablonski [10] proposed a new method for the experimental determination of the inelastic mean free path value of electrons in solids. This method is based on the measurements and theoretical calculations of the so-called coefficient of elastic backscattering of electrons in the solid $-\eta_{\mathrm{e}}$. This coefficient is defined as shown below

$$
\eta_{\mathrm{e}}=I_{\mathrm{el}} / I_{\mathrm{p}}
$$

where $I_{\mathrm{el}}$ is the current of electrons backscattered elastically, $I_{\mathrm{p}}$ - primary electron beam current.

It is clear that the $\eta_{e}$ value for a given element and electron energy can be easily measured by means of any electron energy analyzer because $I_{\mathrm{el}}$ is simply proportional to the area under the so-called elastic peak recorded in secondary electron energy distribution $N(E)=f(E)$.

The theoretical calculations of this coefficient are performed using the Monte Carlo simulation of primary electron trajectory in the solid. The computer randomizes a path of a single electron between successive elastic collisions and azimuthal and polar angles of each elastic scattering. These operations are repeated until an electron leaves the solid and in such a case its contribution to the total current of electrons backscattered elastically $-\mathrm{d} I_{e}$ is calculated in the following way:

$$
\mathrm{d} I_{\mathrm{el}}=\exp \left[\left(-\sum_{i} z_{i}\right) / \lambda\right]
$$

where $\sum_{i} z_{i}$ is the total path of an electron in the solid, $\lambda$ - the assumed value of the inelastic mean free path (if the total path of an electron is higher than about $\left.11 \times \lambda \mathrm{d} I_{\mathrm{el}}=0\right)$.

The result of such calculations is obtained in the form of a histogram as typically shown in Fig. 1. The parameters of a histogram are: the assumed $\lambda$ value and the values of differential cross-sections for elastic scattering of an electron - $(\mathrm{d} \sigma / \mathrm{d} \Omega)_{\theta}(\theta$ is a scattering polar angle) which must be known. In several papers (see for example $[11,12])$ values of $(\mathrm{d} \sigma / \mathrm{d} \Omega)_{\theta}$ were calculated theoretically, however, only for electron scattering on single atoms. The experimental $\lambda$ value is obtained from the comparison of $\eta_{\mathrm{e}}$ measured and calculated theoretically:

$$
\eta_{\mathrm{e}}[\text { measured }]=\eta_{\mathrm{e}}(\lambda)[\text { calculated }] \text {. }
$$




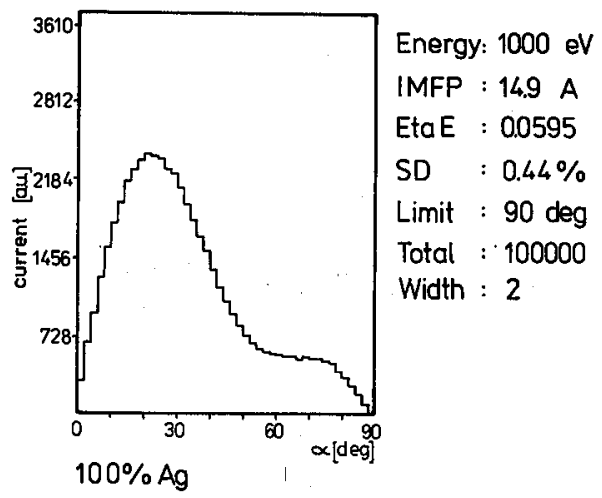

Fig. 1. The typical result of theoretical calculations of the current of electrons elastically backscattered.

The accuracy of the method presented (more in detail it is described in $[10,13,14]$ ) depends on the accuracy of the $\mathrm{d} \sigma / \mathrm{d} \Omega$ calculations because the shape of a histogram presented typically in Fig. 1 depends on the shape of $(\mathrm{d} \sigma / \mathrm{d} \Omega)_{\theta}=f(\theta)$ dependence. So, in order to verify the method of $\lambda$ determination proposed in Ref. [10] the measurements of the angular distribution of the current of electrons backscattered elastically should be performed and compared with the histogram calculated. Such verification was presented by Jabłoński et al. [12], however for two elements only ( $\mathrm{Pd}$ and $\mathrm{Si}$ ) and a few experimental points. In our previous papers $[13,14]$ we have determined $\lambda$ values for $\mathrm{Ag}$ and $\mathrm{Cu}$. Here we present the results of angular measurements of the current of electrons elastically backscattered from polycrystalline silver performed for three values of primary electron energy (250, 500 and $1000 \mathrm{eV}$ ) in the range of emission angles $24^{\circ}-90^{\circ}$ in respect to the surface normal.

\section{Experimental}

All the measurements of angular distributions were performed for polycrystalline silver of high purity (99.99\%). The sample surface was cleaned by in situ heating close to the melting point and the purity was controlled by AES (Auger Electron Spectroscopy). The pressure of rest gases was equal to about $2 \times 10^{-8} \mathrm{~Pa}$. Two different experimental techniques were used for the measurements of angular distributions of secondary electrons and electrons backscattered elastically. Both of them are described below.

\subsection{Secondary (also Auger) electron angular distribution measurements}

For these measurements a small hole $(6 \times 6 \mathrm{~mm})$ was cut in the collector of the typical 4-grid Retarding Field Analyzer (RFA) and an additional collector was mounted behind it as shown in Fig. 2. This way a new, small RFA analyzer was obtained with an acceptance angle equal to $4^{\circ}$. The sample could be rotated in front of this analyzer by means of a rotable manipulator. From the first view 


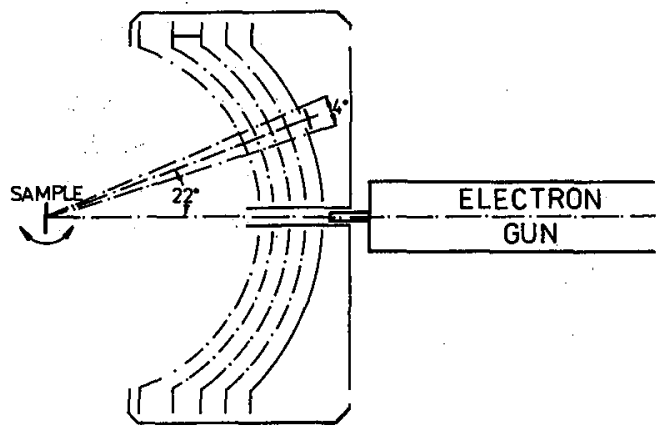

Fig. 2. The system with small collector in RFA analyzer.

this method seems to be not appropriate because while rotating the sample also the incidence angle of the primary electron beam is changed (as it is visible in Fig. 2 an electron gun is mounted along the axis of RFA optics). It.is possible to find however a simple solution of this problem which is presented in Fig. 3. Let us locate the sample in front of the small collector and next rotate it with

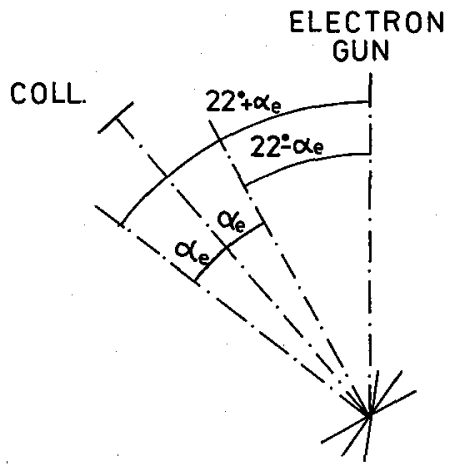

Fig. 3. The experimental procedure for elimination of the influence of the incidence angle on the secondary electron emission.

the same angle $\alpha_{\mathrm{e}}$ left and right with respect to the small collector axis. The measured signal of secondary electrons (or Auger electrons) should be identical for such two sample positions, provided that an incidence angle is the same, because the emission angles are the same (in respect to the sample surface normal) and the sample is polycrystalline. In our measurements these two signals are not identical because the incidence angles are not equal to each other in such two positions. So, the difference between these two signals reflects the signal dependence on the incidence angle. If one measures such pairs of signals for several values of $\alpha_{e}$ one may try to find the form of the signal dependence on the incidence angle with 
quite good accuracy. If this form is known all the measured intensities can be normalized to the same value of the incidence angle. So, the signal dependence on the emission angle can be obtained this way. A typical experimental example of this procedure will be shown in Section 4 .

\subsection{The measurement of angular distributions of electrons elastically backscattered}

The simple procedure of the angular measurements described above could not be used in the case of electrons backscattered elastically because the supposed dependence of the current of these electrons on the incidence angle (connected to some extend with the shape of $\mathrm{d} \sigma / \mathrm{d} \Omega$ on $\theta$ dependence) seems to be rather complicated. For that reason we have built a small RFA analyzer located on the sample manipulator. The applied system of bearings made possible rotations of this analyzer in the range of angles $0^{\circ}-135^{\circ}$ with respect to the sample surface normal. Additionally, the sample could also be rotated in front of the electron gun from $0^{\circ}$ to $60^{\circ}$ measured with respect to the electron gun axis, and this is shown in Fig. 4. Due to the analyzer size for the normal incidence of the primary electron

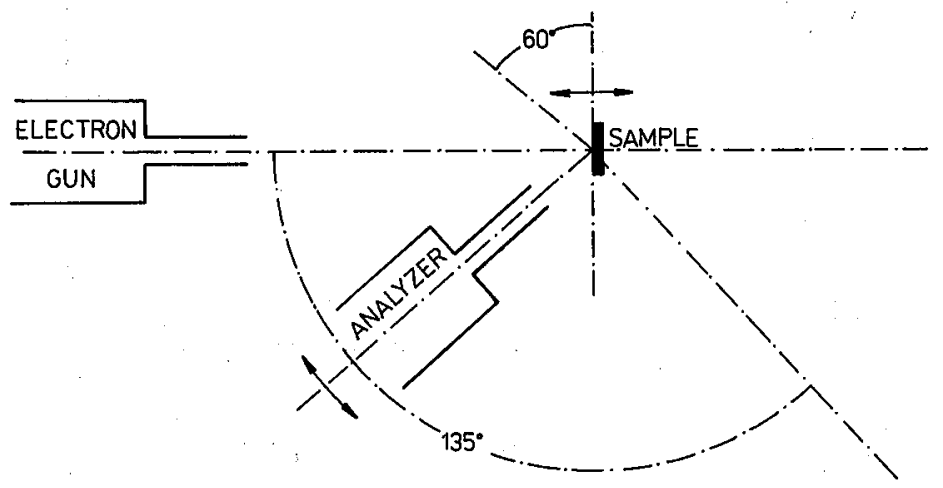

Fig. 4. The scheme of the system with small RFA analyzer.

beam the measurements were possible starting from the emission angle equal to $23^{\circ}$. Instead of the typical collector this analyzer was equipped with a channeltron and its acceptance angle was equal to $3^{\circ}$.

\section{Data acquisition}

The emitted current of secondary electrons of given energy $-e V_{i}$ was recorded in the small collector (see Fig. 2) by applying the constant retarding potential $\left(-V_{\mathrm{i}}\right)$ to the grids of RFA analyzer together with a small modulation voltage equal to $0.56 V_{\mathrm{pp}}$ in all measurements. The whole system was operating in $N(E)=f(E)$ mode and the signal recorded by lock-in nanovoltmeter was taken as a measure for the current of secondary electrons emitted. For a given $V_{i}$ value (i.e. given energy of secondary electrons) the sample was rotated in front of the small collector in 

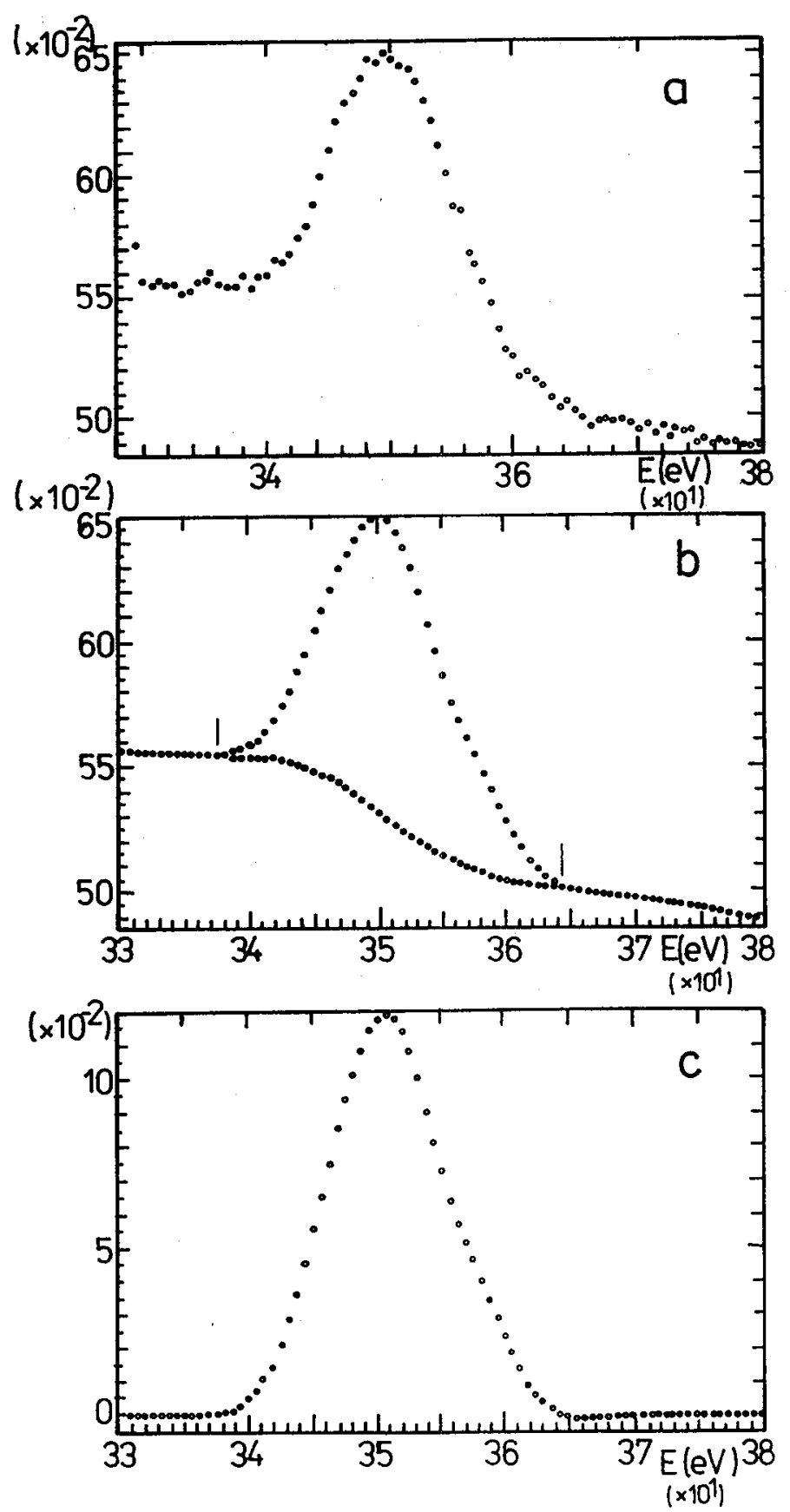

Fig. 5. The typical record of the Ag Auger peak: a) as recorded; b) record after smoothing and background calculations; c) after background subtraction. 

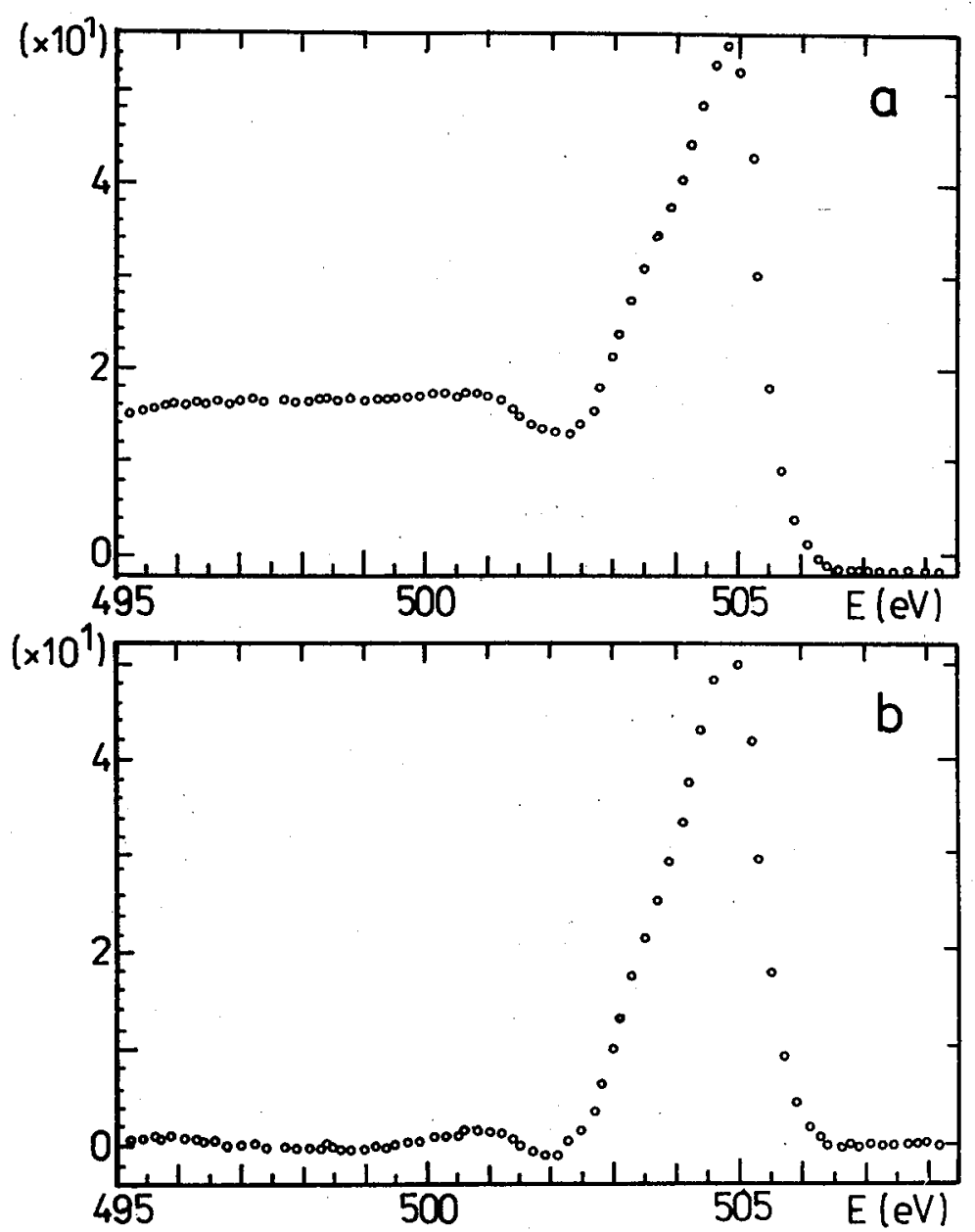

Fig. 6. The typical record of the elastic peak: a) the record; b) after background subtraction.

the range of angles $0^{\circ}-66^{\circ}$ left and $0^{\circ}-88^{\circ}$ right with respect to the small collector axis. Such measurements were performed for secondary electron energies equal to 50,100 and $400 \mathrm{eV}$ and two values of primary electron energy -1000 and $2500 \mathrm{eV}$. The intensities of the Auger (Ag- $M_{2,3} N_{4,5} N_{4,5}$ line) signal was also measured in $N(E)=f(E)$ operating mode for $E_{\mathrm{p}}=2500 \mathrm{eV}, I_{\mathrm{p}}=20 \mu \mathrm{A}$ and $U_{M}=8.4 V_{\mathrm{pp}}$. A typical record is shown in Fig. 5a. In Fig. 5b the same signal is presented after smoothing (cubic spline) and background calculation (Shirley method). The height of the peak shown in Fig. 5 c (after background subtraction) was taken as a measure 
of Auger electron current.

As a measure of the current of electrons backscattered elastically the height of the elastic peak recorded in $N(E)=f(E)$ mode by means of the small rotatable RFA analyzer was taken. A typical record of this peak obtained for $E_{\mathrm{p}}=505 \mathrm{eV}$ is shown in Fig. 6. As it is visible the resolution of this analyzer is equal to about $0.32 \%$. The modulation voltage in these measurements was equal to $0.4 V_{\mathrm{pp}}$.

\section{Results and discussion}

The most important problem in the first part of our measurements was to find the form of the dependence of the measured secondary and Auger signals on the primary beam incidence angle. As it was described in Section 2.1 for each $\alpha_{e}$ angle (see Fig. 2) two signals were measured with different incidence angles $\alpha_{i}-\alpha_{i}^{1}=22^{\circ}+\alpha_{\mathrm{e}}$ and $\alpha_{i}^{2}=22^{\circ}-\alpha_{\mathrm{e}}$. The values of these signals recorded for a secondary electron energy of $50 \mathrm{eV}$, a primary energy $E_{\mathrm{p}}=2500 \mathrm{eV}$ and for three $\alpha_{\mathrm{e}}$ values are shown below ( $I-$ intensity):

$\begin{array}{ccc}\alpha_{\mathrm{e}} & \alpha_{i} & I \text { [arb. units] } \\ 15^{\circ} & 37^{\circ} & 0.1156 \\ & 7^{\circ} & 0.0976 \\ 10^{\circ} & 32^{\circ} & 0.1321 \\ & 12^{\circ} & 0.1102 \\ 5^{\circ} & 27^{\circ} & 0.134 \\ & 17^{\circ} & 0.1181\end{array}$

Based on such measurements for 10 or $12 \alpha_{\mathrm{e}}$ values for different energies of secondary electrons (also for Auger electrons) we found that the signals measured for a given $\alpha_{\mathrm{e}}$ become equal to each other with an accuracy of about $5 \%$, if their intensities are multiplied by the cosine of the incidence angle. An example is given below:

$\begin{array}{rrcc}\alpha_{\mathrm{e}} & \alpha_{i} & I \text { [arb. units] } & I \times \cos \alpha_{i} \\ 15^{\circ} & 37^{\circ} & 0.1156 & 0.0923 \\ & 7^{\circ} & 0.0976 & 0.0929 \\ 10^{\circ} & 32^{\circ} & 0.1321 & 0.1120 \\ & 12^{\circ} & 0.1102 & 0.1078 \\ 5^{\circ} & 27^{\circ} & 0.1341 & 0.1197 \\ & 17^{\circ} & 0.1181 & 0.1130\end{array}$

It simply means that both secondary and Auger yields are inversely proportional to the cosine of the incidence angle. So, the multiplication of a given signal by the cosine of the incidence angle gives the signal normalization to the incidence angle of $0^{\circ}$ with respect to the surface normal according to the equation given below:

$$
I_{1}\left(\alpha_{\mathrm{e}}, \alpha_{i}^{1}\right) \cos \alpha_{i}^{1}=I_{2}\left(\alpha_{\mathrm{e}}, \alpha_{i}^{2}\right) \cos \alpha_{i}^{2}=I_{3}\left(\alpha_{\mathrm{e}}, \alpha_{i}^{3}\right) \cos \alpha_{i}^{3}=I_{0}\left(\alpha_{\mathrm{e}}\right) \text { if } \alpha_{i}=0^{\circ} \text {. (3) }
$$

The real angular distributions of Auger and secondary electrons emissions obtained in the above way are presented in Figs. 7-9. As it is visible all of them are of the cosine-type. 


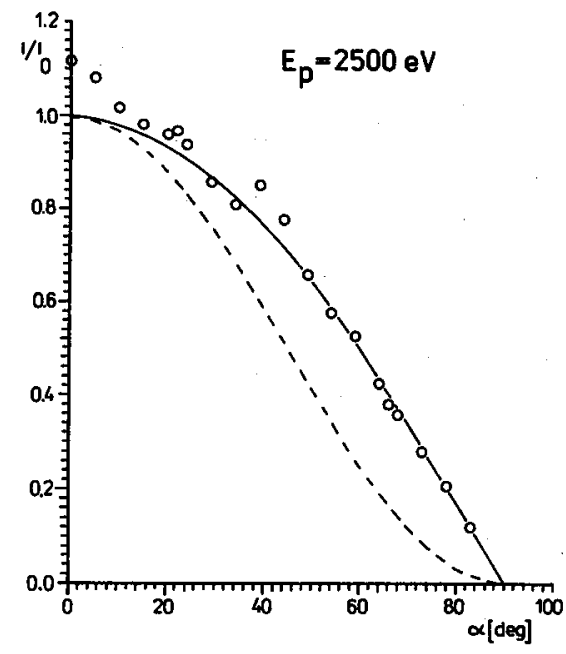

Fig. 7. Angular distribution of Auger electron emission; (o - experimental results, solid and dashed lines - cosine and cosine square distribution, respectively); $I_{0}$ - mean value of $\left(I^{k} \cos \alpha_{i}^{k} / \cos \alpha_{\mathrm{e}}^{k}\right)$.
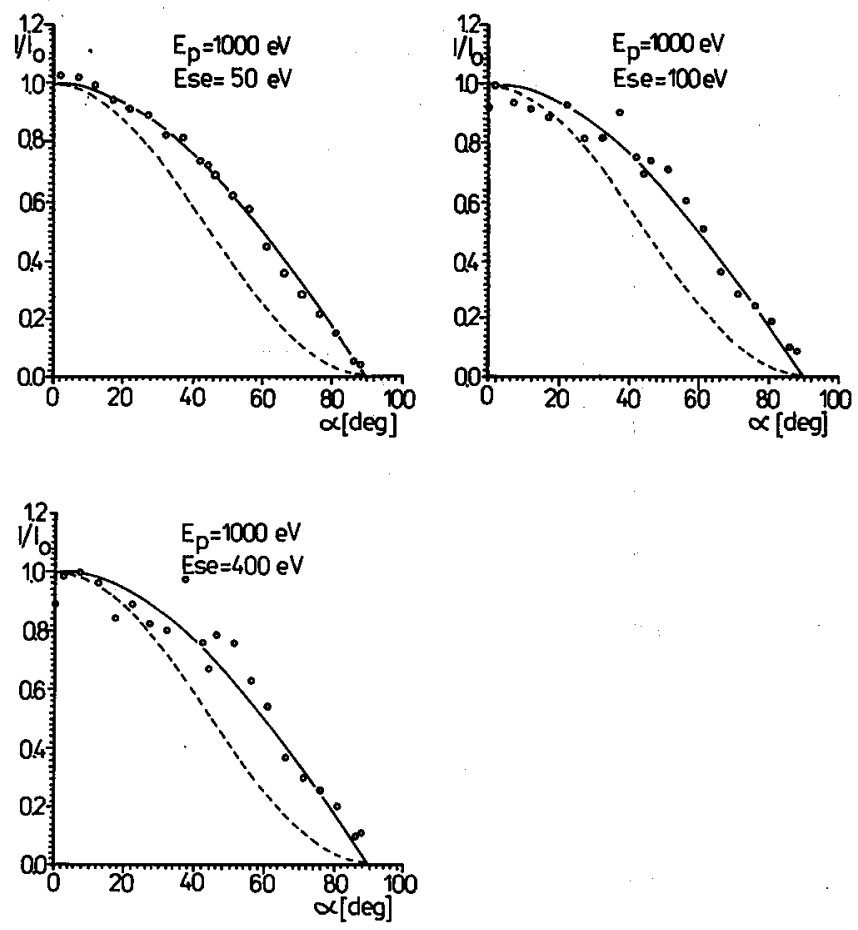

Fig. 8. Angular distribution of sccondary electron emission $-E_{\mathrm{p}}=1000 \mathrm{eV}$; (o experimental results, solid and dashed lines - cosine and cosine square distribution, respectively); $I_{0}-$ mean value of $\left(I^{k} \cos \alpha_{i}^{k} / \cos \alpha_{e}^{k}\right)$. 

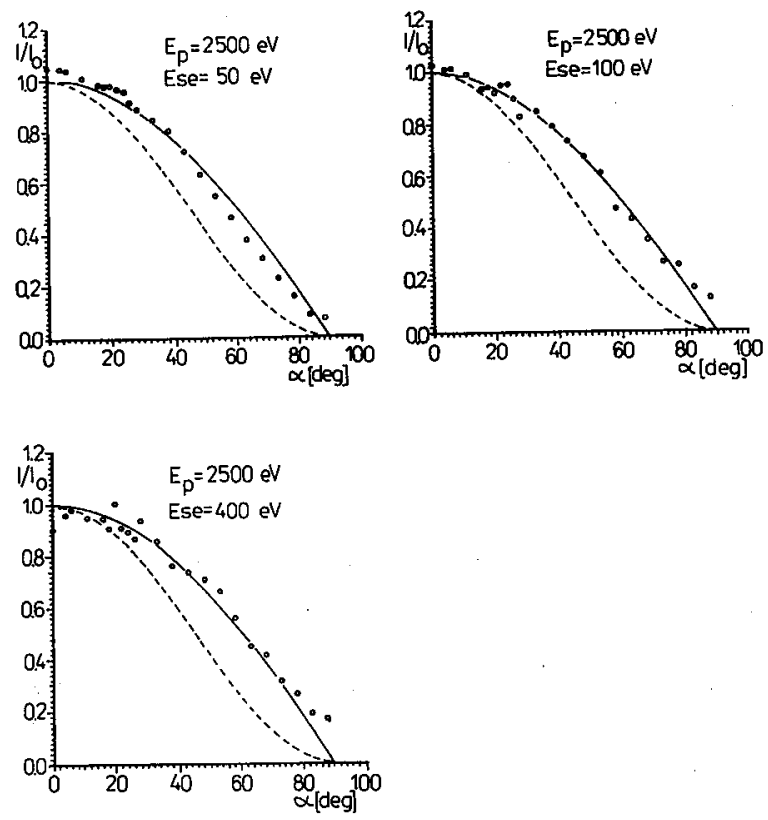

Fig. 9. Angular distribution of secondary electron emission $-E_{\mathrm{p}}=2500 \mathrm{eV}$; ( experimental results, solid and dashed lines - cosine and cosine square distribution, respectively); $I_{0}-$ mean value of $\left(I^{k} \cos \alpha_{i}^{k} / \cos \alpha_{\mathrm{e}}^{k}\right)$.

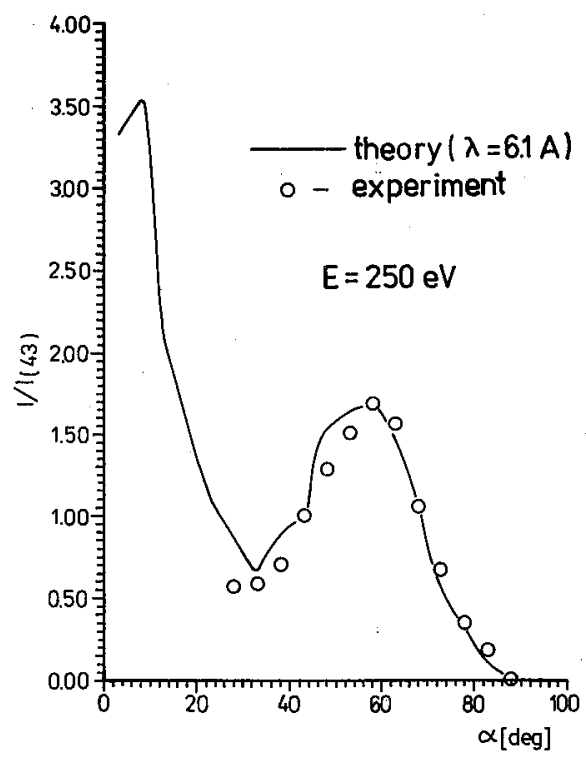

Fig. 10. Angular distribution of electrons backscattered elastically, $E_{\mathrm{p}}=250 \mathrm{eV} ; I(43)$ $-I$ value for $\alpha_{\mathrm{e}}=43^{\circ}$. 
In Figs. 10-12 the angular distributions of electrons backscattered elastically measured with the small rotable RFA analyzer are shown for three values of primary electron energy. The solid line in each figure represents the elastic cur-
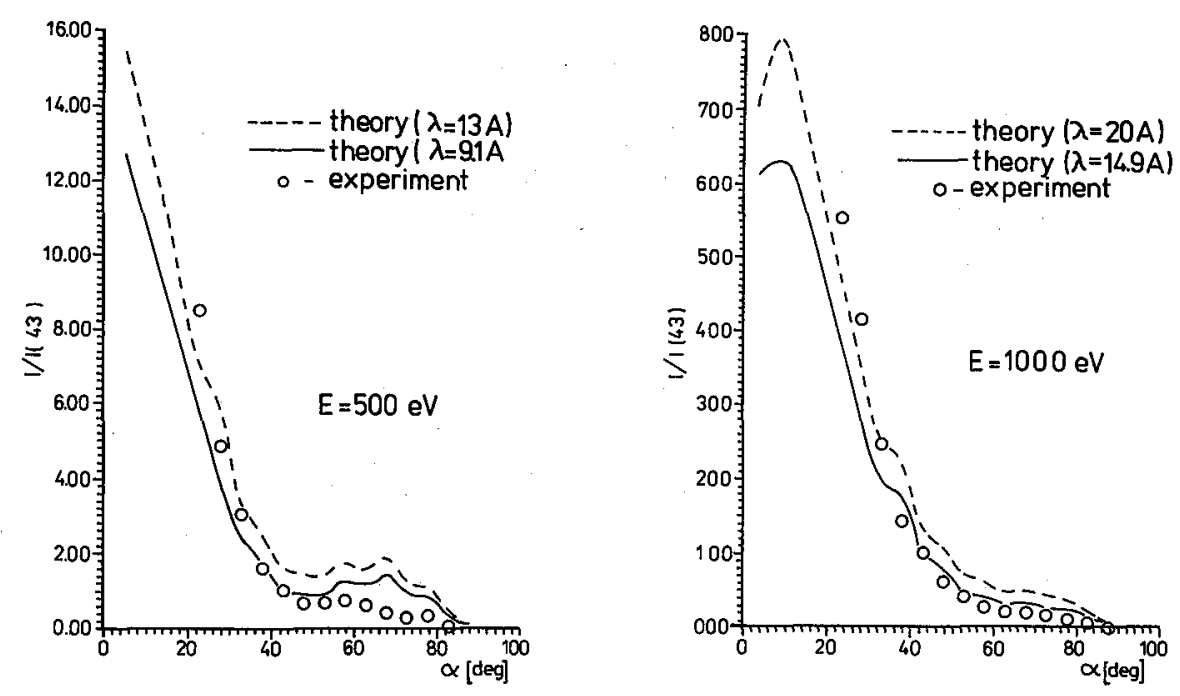

Fig. 11. Angular distribution of electrons backscattered elastically, $E_{\mathrm{p}}=500 \mathrm{eV} ; I(43)$ $-I$ value for $\alpha_{\mathrm{e}}=43^{\circ}$.

Fig. 12. Angular distribution of electrons backscattered elastically, $E_{\mathrm{p}}=1000 \mathrm{eV}$; $I(43)-I$ value for $\alpha_{e}=43^{\circ}$.

rent calculated theoretically (Monte Carlo simulation) where the $(\mathrm{d} \sigma / \mathrm{d} \Omega)_{\theta}$ values were taken from the paper of Fink and Ingram [11]. As one can see in Fig. 10 $\left(E_{\mathrm{p}}=250 \mathrm{eV}\right)$ the agreement between theoretical calculations and the measurements is quite good. For higher energies of primary electrons some discrepancies are observed. For these energies two histograms were calculated for different assumed $\lambda$ values (solid and dashed lines in Figs. 11 and 12). The results obtained for higher $\lambda$ 's (dashed lines) were normalized to the $I\left(43^{\circ}\right)$ value obtained for lower $\lambda$ 's to show the increase of the elastic current calculated theoretically with the increase of $\lambda$. As one can see the increase of $\lambda$ in calculations gives a better agreement between theory and experiment for lower emission angles; however, in such a case the difference for higher angles increases. This suggests that the values of $(\mathrm{d} \sigma / \mathrm{d} \Omega)_{\theta}$ are not quite correct for higher electron energies.

\section{Conclusions}

The presented results show the cosine-type angular distribution both for Auger and secondary electron emission independent on secondary electron energy. In addition, the intensities of these emissions are inversely related to the cosine of the incidence angle of the primary electron beam. 
The angular distribution of electrons backscattered elastically depends on electron energy. The good agreement between intensities of elastic current measured experimentally and calculated suggests the correctness of the Monte Carlo algorithm proposed by Jabłoński [10] for $\lambda$ determination.

\section{Acknowledgements}

This work was supported by Polish Ministry of National Education (grant DNS-P/04/097/90-2). The construction of the system for the angular measurements was supported by University of Wroclaw (grant 10.10/S/IFD/91).

\section{References}

[1] J. Palczyński, W. Doliński, S. Mróz, Surf. Sci. 247, 395 (1991).

[2] R.L. Gerlach, A.R. Ducharme, Surf. Sci. 32, 329 (1972).

[3] J.L.H. Jonker, Philips Res. Repts. 6, 372 (1951); Philips Res. Repts. 7,1 (1952).

[4] H. Bruining, Physics and Applications of Secondary Electron Emission, Pergamon Press, London 1954.

[5] H. Kanter, Ann. Physik 20, 144 (1957).

[6] J.B. Pendry, J. Phys. C 8, 2413 (1975).

[7] D. Aberdam, R. Baudoing, E. Blanc, C. Gaubert, Surf. Sci. 57, 306 (1976).

[8] R. Shimizu, S. Ichimura, Quantitative Analysis by Auger Electron Spectroscopy, Technical Reports Toyota Foundation No. I-006, 76-0175, Toyota Foundation, Tokyo 1981.

[9] T. Oguri, H. Islioka, H. Fukuda, M. Irako, J. Phys. Soc. Jpn. 55, 414 (1986).

[10] A. Jabłoński, Surf. Sci. 151, 166 (1985).

[11] M. Fink, D. Ingram, At. Data Nucl. Data Tables 4, 129 (1972).

[12] A. Jabłoński, J. Gryko, J. Kraaer, S. Tougaard, Phys. Rev. B 39, 61 (1989).

[13] W. Doliński, H. Nowicki, S. Mróz, Surf. Interface Anal. 11, 229 (1988).

[14] W. Doliński S. Mróz, M. Zagórski, Surf. Sci. 200, 361 (1988). 